Supporting Information

\title{
Enhanced Photooxidation of Hydroquinone by Acetylacetone, a Novel Photosensitizer and Electron Shuttle
}

Jiyuan Jin ${ }^{1}$, Shujuan Zhang ${ }^{*}$, Bingdang $\mathrm{Wu}^{1}$, Zhihao Chen $^{1}$, Guoyang Zhang ${ }^{1}$, and Paul G. Tratnyek $^{2}$

${ }^{1}$ State Key Laboratory of Pollution Control and Resource Reuse, School of the Environment, Nanjing University, Nanjing, 210023, China

${ }^{2}$ OHSU-PSU School of Public Health, Oregon Health \& Science University, 3181 SW Sam Jackson Park Road, Portland, OR 97239, USA

*Correspondence author. Phone: +86258968 0389, E-mail: sjzhang@nju.edu.cn

Submitted to: Environmental Science \& Technology

This file contains 5 Figures and 3 Tables. 


\section{Figure and Table Captions}

Figure S1. The UV absorption spectra of the $\mathrm{QH}_{2}$-AA binary solution immediately and 6-h later after mixing at ambient condition ( $\mathrm{pH}: 6.0$, indoor lighting, room temperature).

Figure S2. The conversion profiles of $\mathrm{QH}_{2}$ in buffered and unbuffered $\mathrm{QH}_{2}-\mathrm{AA}$ binary solutions. $[\mathrm{AA}]_{0}$ and $\left[\mathrm{QH}_{2}\right]_{0}: 0.2 \mathrm{mM}$, initial $\mathrm{pH}: 6.5, \mathrm{UV}: 5.3 \mathrm{~mW} \cdot \mathrm{cm}^{-2}$ at $365 \mathrm{~nm}$.

Figure S3. (a) The absorption spectra of $\mathrm{QH}_{2}, \mathrm{AA}$, and the mixture of $\mathrm{QH}_{2}$ and AA. (b) The absorption spectra of the raw and UV irradiated $\mathrm{QH}_{2}$-AA solutions. (c) The fraction of available photons to AA in the raw and UV irradiated $\mathrm{QH}_{2}-\mathrm{AA}$ solutions. The dashed spectra in (a) and (b) are the spectral irradiance of the light source used in the photochemical experiments. $[\mathrm{AA}]_{0}=[\mathrm{QH}]_{0}=0.5 \mathrm{mM}, \mathrm{UV}: 3.9 \mathrm{~mW} \cdot \mathrm{cm}^{-2}$ at $365 \mathrm{~nm}$.

Figure S4. The total ion current spectra of $\mathrm{QH}_{2}$ in the absence (left) and presence (right) of AA after 30 min UV irradiation. $\left[\mathrm{QH}_{2}\right]_{0}=[\mathrm{AA}]_{0}=0.5 \mathrm{mM}, \mathrm{UV}: 4.3 \mathrm{~mW} \cdot \mathrm{cm}^{-2}$ at $365 \mathrm{~nm}$.

Figure S5. The transient species generated in photolyzed $\mathrm{QH}_{2}(1 \mathrm{mM})$ solution. (a) $e_{\mathrm{aq}}{ }^{-}$and $\cdot \mathrm{OH}$ with DMPO (100 mM) as the probing molecule; (b) $\mathrm{Q}^{{ }^{-}}$, without probing molecule.

Table S1. The consumption rate constant of $\mathrm{O}_{2}$ and the formation rate constant of $\mathrm{H}_{2} \mathrm{O}_{2}$ in UV irradiated $\mathrm{QH}_{2}, \mathrm{QH}_{2}-\mathrm{AA}$, and AA solutions. $\left[\mathrm{QH}_{2}\right]_{0}=[\mathrm{AA}]_{0}=0.2 \mathrm{mM}, \mathrm{UV}: 6.0$ $\mathrm{mW} \cdot \mathrm{cm}^{-1}$ at $365 \mathrm{~nm}$.

Table S2. Identified products in UV irradiated $\mathrm{QH}_{2}$ (the first six compounds) and $\mathrm{QH}_{2}-\mathrm{AA}$ solutions.

Table S3. The ion current intensity of the identified $\mathrm{QH}_{2}$ products in Table $\mathrm{S} 2$. 


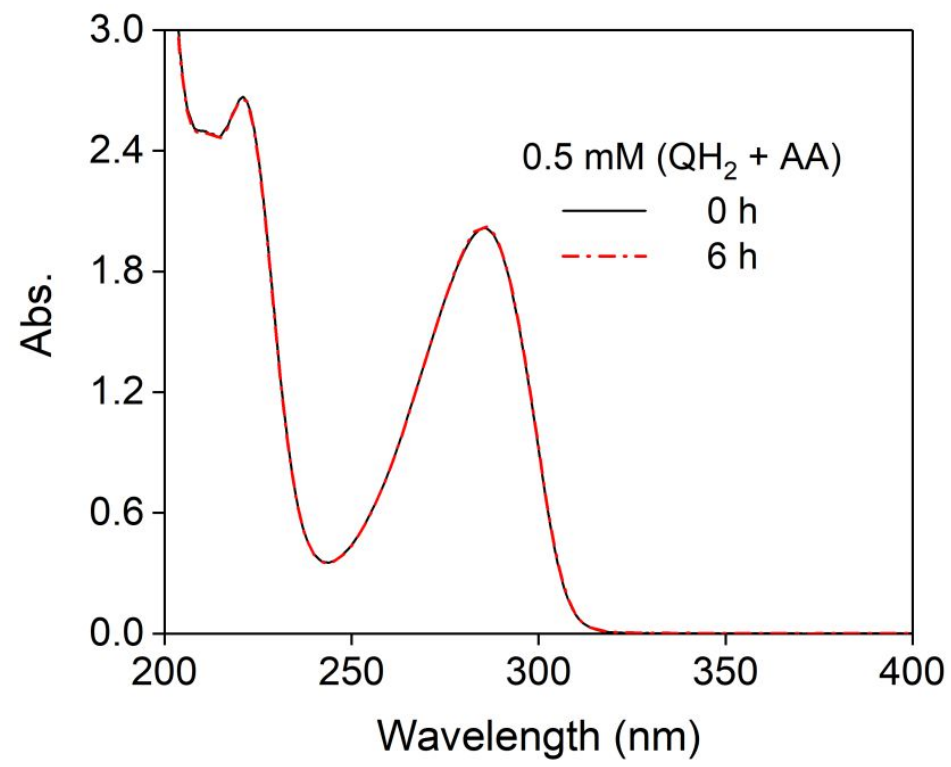

Figure S1. The UV absorption spectra of the $\mathrm{QH}_{2}$-AA binary solution immediately and 6-h after mixing at ambient condition (pH: 6.0, indoor lighting, room temperature). 


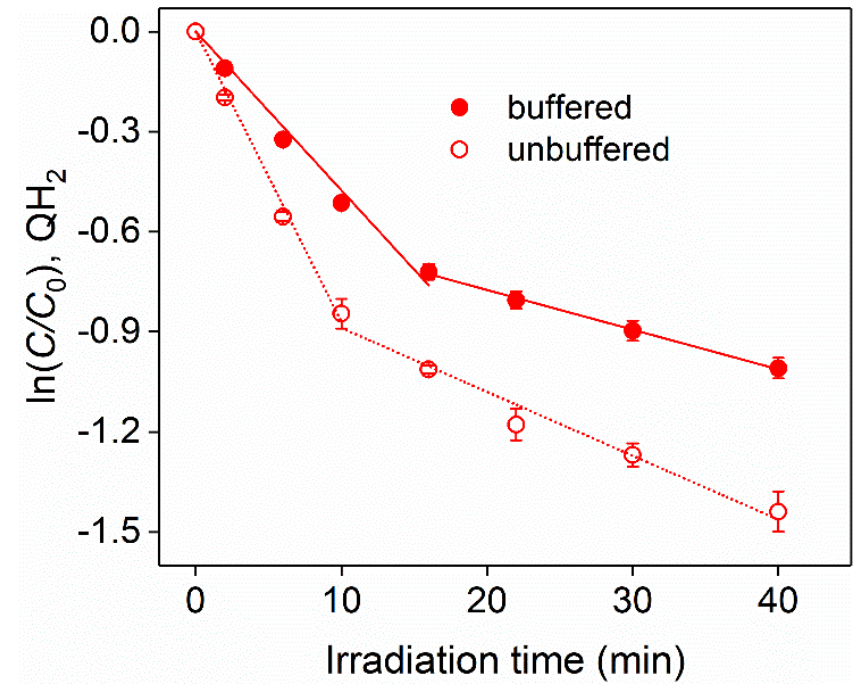

Figure S2. The conversion profiles of $\mathrm{QH}_{2}$ in buffered and unbuffered $\mathrm{QH}_{2}-\mathrm{AA}$ binary solutions.

$[\mathrm{AA}]_{0}$ and $\left[\mathrm{QH}_{2}\right]_{0}: 0.2 \mathrm{mM}$, initial $\mathrm{pH}: 6.5, \mathrm{UV}: 5.3 \mathrm{~mW} \cdot \mathrm{cm}^{-2}$ at $365 \mathrm{~nm}$. 

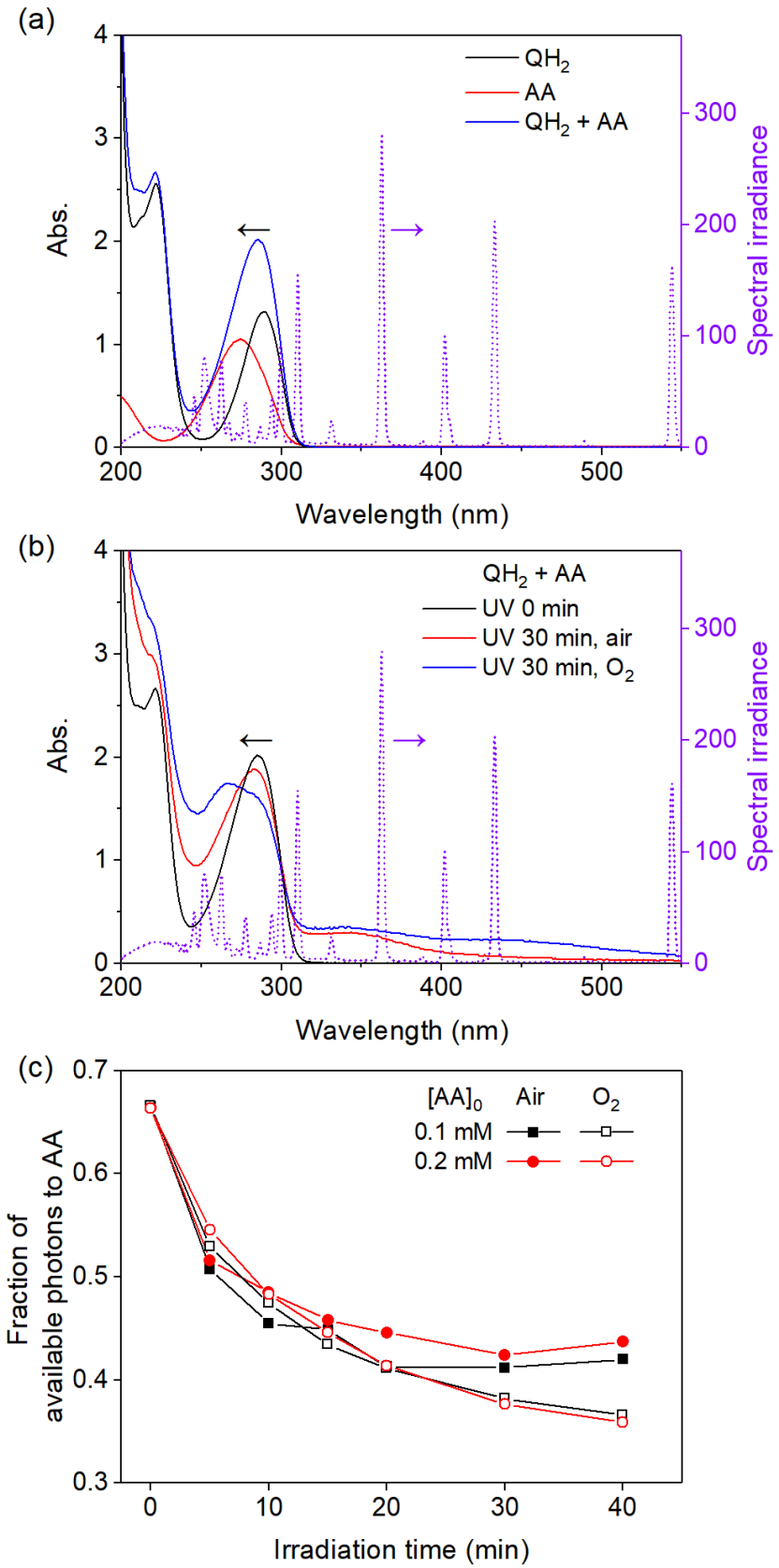

Figure S3. (a) The absorption spectra of $\mathrm{QH}_{2}, \mathrm{AA}$, and the mixture of $\mathrm{QH}_{2}$ and $\mathrm{AA}$. (b) The absorption spectra of the raw and UV irradiated $\mathrm{QH}_{2}-\mathrm{AA}$ solutions. (c) The fraction of available photons to AA in the raw and UV irradiated $\mathrm{QH}_{2}-\mathrm{AA}$ solutions. The dashed spectra in (a) and (b) are the spectral irradiance of the light source used in the photochemical experiments. $[\mathrm{AA}]_{0}=$ $\left[\mathrm{QH}_{2}\right]_{0}=0.5 \mathrm{mM}, \mathrm{UV}: 3.9 \mathrm{~mW} \cdot \mathrm{cm}^{-2}$ at $365 \mathrm{~nm}$. 

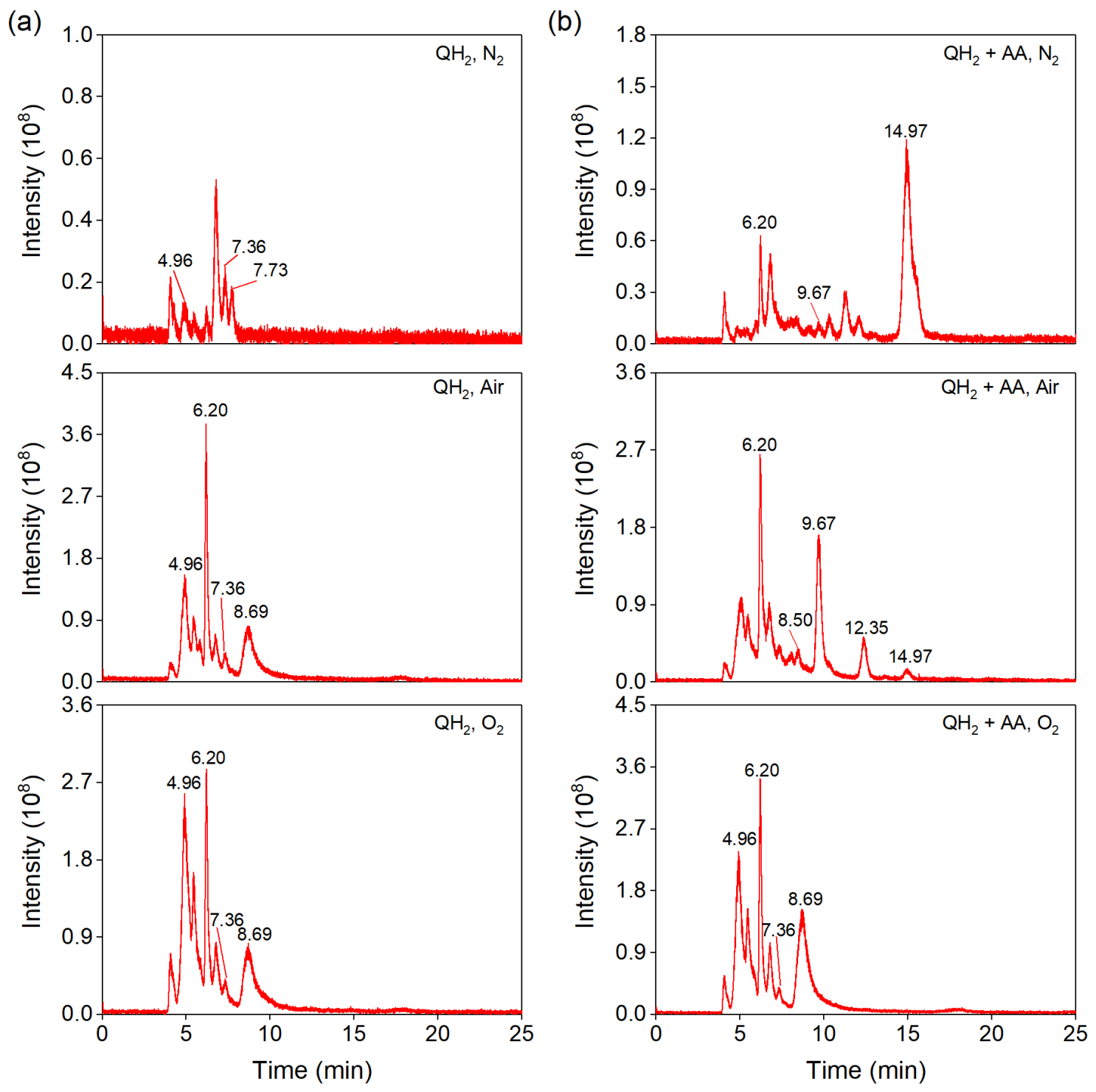

Figure S4. The total ion current spectra of $\mathrm{QH}_{2}$ in the absence (left) and presence (right) of AA after $30 \mathrm{~min} \mathrm{UV}$ irradiation. $\left[\mathrm{QH}_{2}\right]_{0}=[\mathrm{AA}]_{0}=0.5 \mathrm{mM}, \mathrm{UV}: 4.3 \mathrm{~mW} \cdot \mathrm{cm}^{-2}$ at $365 \mathrm{~nm}$. 

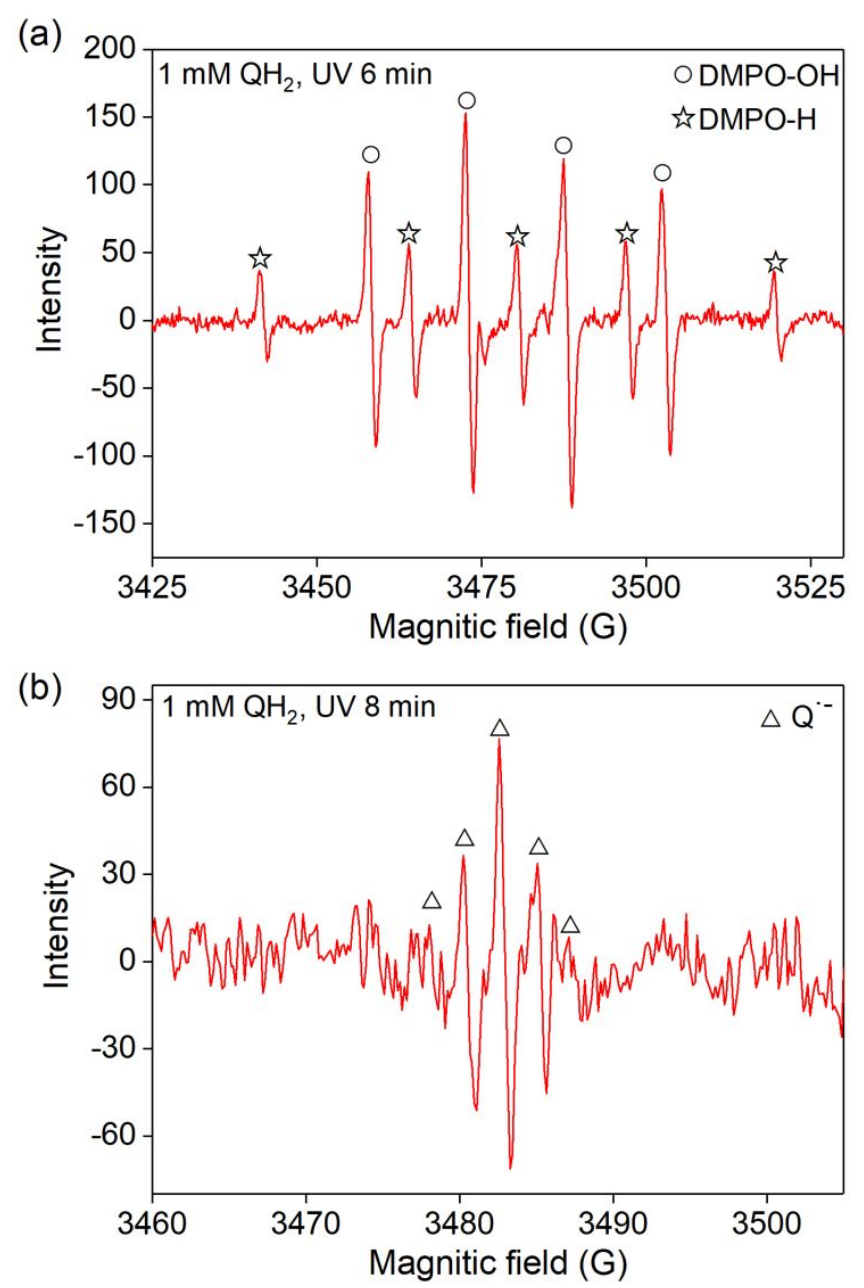

Figure S5. The transient species generated in photolyzed $\mathrm{QH}_{2}(1 \mathrm{mM})$ solution. (a) $e_{\mathrm{aq}}{ }^{-}$and $\cdot \mathrm{OH}$ with DMPO (100 mM) as the probing molecule; (b) $\mathrm{Q}^{*^{-}}$, without probing molecule. 
Table S1. The consumption rate constant of $\mathrm{O}_{2}$ and the formation rate constant of $\mathrm{H}_{2} \mathrm{O}_{2}$ in UV irradiated $\mathrm{QH}_{2}, \mathrm{QH}_{2}-\mathrm{AA}$, and AA solutions. $\left[\mathrm{QH}_{2}\right]_{0}=[\mathrm{AA}]_{0}=0.2 \mathrm{mM}, \mathrm{UV}: 6.0 \mathrm{~mW} \cdot \mathrm{cm}^{-1}$ at 365 nm.

\begin{tabular}{lllllllll}
\hline & \multicolumn{3}{c}{$\mathrm{Air}$} & & & \multicolumn{3}{c}{$\mathrm{O}_{2}$} \\
\cline { 2 - 4 } & $\mathrm{QH}_{2}$ & $\mathrm{QH}_{2}-\mathrm{AA}$ & $\mathrm{AA}$ & & & $\mathrm{QH}_{2}$ & $\mathrm{QH}_{2}-\mathrm{AA}$ & $\mathrm{AA}$ \\
\hline$k_{1, \mathrm{O}_{2}}\left(\mathrm{~min}^{-1}\right)$ & 0.081 & 0.133 & 0.126 & & $\mathrm{ND}^{a}$ & $\mathrm{ND}$ & $\mathrm{ND}$ \\
$k_{0, \mathrm{H}_{2} \mathrm{O}_{2}}(\mu \mathrm{M} / \mathrm{min})$ & 1.542 & 1.288 & 1.256 & & 3.601 & 1.810 & 0.642 \\
\hline
\end{tabular}

${ }^{a}$ not detected. 
Table S2. Identified products in UV irradiated $\mathrm{QH}_{2}$ (the first six compounds) and $\mathrm{QH}_{2}-\mathrm{AA}$ solutions.

\begin{tabular}{|c|c|c|c|c|}
\hline Symbol & $\begin{array}{l}\text { Molecular } \\
\text { formula }\end{array}$ & Chemical structure & $\begin{array}{l}\text { Retention } \\
\text { time (min) }\end{array}$ & $\begin{array}{c}\text { Mass charge ratio } \\
(\mathrm{m} / \mathrm{z})\end{array}$ \\
\hline QOH & $\mathrm{C}_{6} \mathrm{H}_{4} \mathrm{O}_{3}$ & & 7.36 & 123.00712 \\
\hline THBP & $\mathrm{C}_{12} \mathrm{H}_{10} \mathrm{O}_{4}$ & & 7.73 & 217.04923 \\
\hline $\mathrm{M}_{1}$ & $\mathrm{C}_{12} \mathrm{H}_{8} \mathrm{O}_{6}$ & & 6.20 & 247.02350 \\
\hline $\mathrm{M}_{2}$ & $\mathrm{C}_{12} \mathrm{H}_{10} \mathrm{O}_{6}$ & & 5.83 & 249.03926 \\
\hline $\mathrm{M}_{3}$ & $\mathrm{C}_{12} \mathrm{H}_{8} \mathrm{O}_{7}$ & & 8.69 & 263.01847 \\
\hline $\mathrm{M}_{4}$ & $\mathrm{C}_{12} \mathrm{H}_{10} \mathrm{O}_{7}$ & & 4.96 & 265.03427 \\
\hline $\mathrm{QH}_{2}-\mathrm{C}_{2} \mathrm{H}_{3} \mathrm{O}$ & $\mathrm{C}_{8} \mathrm{H}_{8} \mathrm{O}_{3}$ & & 14.97 & 151.03841 \\
\hline $\begin{array}{l}\mathrm{CH}_{3}-\mathrm{QH}_{2}^{-} \\
\mathrm{C}_{2} \mathrm{H}_{3} \mathrm{O}\end{array}$ & $\mathrm{C}_{9} \mathrm{H}_{10} \mathrm{O}_{3}$ & & 8.09 & 165.05409 \\
\hline
\end{tabular}




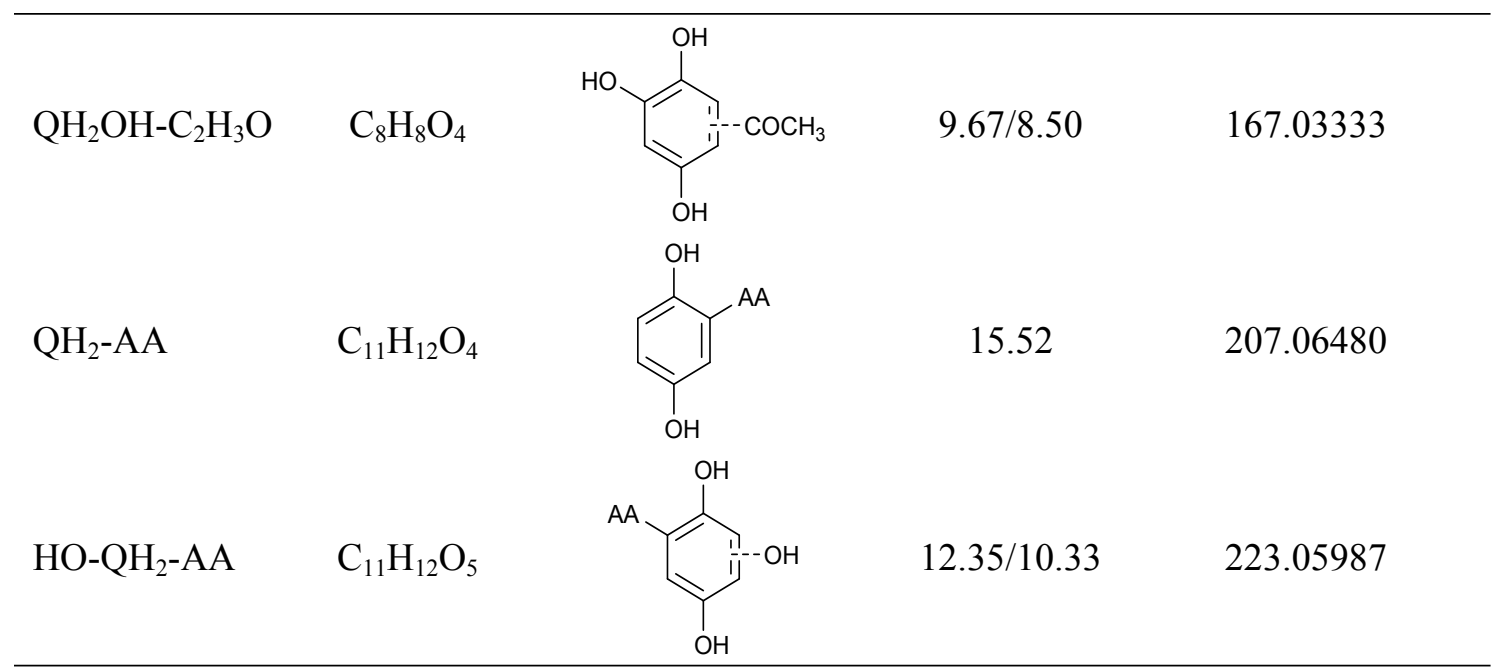


Table S3. The ion current intensity of the identified $\mathrm{QH}_{2}$ products in Table S2.

\begin{tabular}{|c|c|c|c|c|c|c|c|}
\hline \multirow{2}{*}{ Products } & \multirow{2}{*}{$\begin{array}{l}\text { UV time } \\
\text { (min) }\end{array}$} & \multicolumn{3}{|c|}{$\mathrm{w} / \mathrm{o} \mathrm{AA}$} & \multicolumn{3}{|c|}{$\mathrm{w} / \mathrm{AA}$} \\
\hline & & Air & $\mathrm{O}_{2}$ & $\mathrm{~N}_{2}$ & Air & $\mathrm{O}_{2}$ & $\mathrm{~N}_{2}$ \\
\hline \multirow[t]{2}{*}{$\mathrm{QOH}$} & 15 & $1.51 \mathrm{E}+7$ & $1.46+\mathrm{E} 7$ & $9.51+\mathrm{E} 6$ & $1.25 \mathrm{E}+7$ & $1.21 \mathrm{E}+7$ & $1.42 \mathrm{E}+7$ \\
\hline & 30 & $2.08 \mathrm{E}+7$ & $1.46+\mathrm{E} 7$ & $1.16 \mathrm{E}+7$ & $1.20 \mathrm{E}+7$ & $1.60 \mathrm{E}+7$ & $1.47 \mathrm{E}+6$ \\
\hline \multirow[t]{2}{*}{ THBP } & 15 & $2.11 \mathrm{E}+6$ & $1.39 \mathrm{E}+6$ & $6.96 \mathrm{E}+6$ & $1.05 \mathrm{E}+6$ & $2.32 \mathrm{E}+6$ & $* b$ \\
\hline & 30 & $2.43 \mathrm{E}+6$ & $1.69 \mathrm{E}+6$ & $8.26 \mathrm{E}+6$ & $1.05 \mathrm{E}+6$ & $2.04 \mathrm{E}+6$ & $*$ \\
\hline \multirow[t]{2}{*}{$\mathrm{M}_{1}$} & 15 & $2.59 \mathrm{E}+7$ & $1.23 \mathrm{E}+7$ & $x^{a}$ & $9.26 \mathrm{E}+6$ & $2.55 \mathrm{E}+7$ & $x$ \\
\hline & 30 & $2.55 \mathrm{E}+7$ & $2.16 \mathrm{E}+7$ & $x$ & $2.45 \mathrm{E}+6$ & $3.40 \mathrm{E}+7$ & $x$ \\
\hline \multirow[t]{2}{*}{$\mathrm{M}_{2}$} & 15 & $2.19 \mathrm{E}+7$ & $2.88 \mathrm{E}+6$ & $x$ & $6.34 \mathrm{E}+6$ & $1.41 \mathrm{E}+7$ & $x$ \\
\hline & 30 & $1.81 \mathrm{E}+7$ & $8.19 \mathrm{E}+6$ & $x$ & $1.66 \mathrm{E}+6$ & $1.36 \mathrm{E}+7$ & $x$ \\
\hline \multirow[t]{2}{*}{$\mathrm{M}_{3}$} & 15 & $3.43 \mathrm{E}+7$ & $2.77 \mathrm{E}+7$ & $x$ & $1.36 \mathrm{E}+7$ & $6.97 \mathrm{E}+7$ & $\times$ \\
\hline & 30 & $3.85 \mathrm{E}+7$ & $3.45 \mathrm{E}+7$ & $x$ & $8.11 \mathrm{E}+5$ & $7.66 \mathrm{E}+7$ & $x$ \\
\hline \multirow[t]{2}{*}{$\mathrm{M}_{4}$} & 15 & $2.29 \mathrm{E}+7$ & $2.60 \mathrm{E}+7$ & $1.10 \mathrm{E}+6$ & $1.02 \mathrm{E}+7$ & $3.81 \mathrm{E}+7$ & $x$ \\
\hline & 30 & $2.46 \mathrm{E}+7$ & $2.14 \mathrm{E}+7$ & $1.42 \mathrm{E}+6$ & $3.66 \mathrm{E}+6$ & $3.23 \mathrm{E}+7$ & $x$ \\
\hline
\end{tabular}

$a$ " $\times "$ means undetectable.

$b$ “*” means very weak. 